\section{$\underset{\substack{\text { hommes } \\ \text { \& migrations }}}{ }$}

\section{Hommes \& migrations}

Revue française de référence sur les dynamiques

migratoires

$1315 \mid 2016$

Ondes de choc

\title{
Les attentats comme objets médiatiques instables
}

Une enquête sous l'angle des humanités numériques

\section{Diego Landivar, Émilie Ramillien et Marco Dell'Omodarme}

\section{OpenEdition \\ 1 Journals}

Édition électronique

URL : http://journals.openedition.org/hommesmigrations/3708

DOI : $10.4000 /$ hommesmigrations.3708

ISSN : 2262-3353

Éditeur

Musée national de l'histoire de l'immigration

Édition imprimée

Date de publication : 1 juillet 2016

Pagination : 19-30

ISBN : 978-2-919040-36-0

ISSN : 1142-852X

Référence électronique

Diego Landivar, Émilie Ramillien et Marco Dell'Omodarme, « Les attentats comme objets médiatiques instables », Hommes \& migrations [En ligne], 1315 | 2016, mis en ligne le 01 juillet 2019, consulté le 02 janvier 2020. URL : http://journals.openedition.org/hommesmigrations/3708 ; DOI : 10.4000/

hommesmigrations.3708 


\section{LES ATTENTATS COMME OBJETS MÉDIATIQUES INSTABLES}

\section{UNE ENQUÊTE SOUS L'ANGLE DES HUMANITÉS NUMÉRIQUES}

Par DIEGO LANDIVAR, enseignant-chercheur en humanités numériques (ESC Clermont), directeur d'Origens Medialab, ÉMILIE RAMILLIEN, anthropologue, co-fondatrice d'Origens Medialab, et MARCO DELL'OMODARME, maître de conférences en philosophie et théories de la culture (Institut ACTE), université Paris-1 Panthéon-Sorbonne, chercheur associé à Origens Medialab.

Figure anxiogène et pathétique, le terroriste est un sujet de choix pour les médias français depuis la multiplication des attentats dans I'Hexagone en 2015. Si ses actes sont soumis à la sanction juridique, son profil reste instable et sa fluctuation nourrit de multiples discours. Le terroriste apparaît au centre d'un processus d'objectivation médiatique, comme un objet construit par les médias. L'étude des stratégies discursives et des champs sémantiques mobilisés dans la presse française permet d'interroger le mode d'existence attribué au «terroriste ».

Comment nommer avec justesse ce qui nous est arrivé? Les attentats de janvier et de novembre 2015 ont créé un certain nombre de déplacements symboliques, sociaux et politiques qui paraissent difficilement identifiables dans la mesure où, politiques, médias, scientifiques semblent englués dans le récit des événements. Le cadre de lecture imposé par le script du terrorisme nous place dans une position scientifique inconfortable dans la mesure où il renvoie immédiatement à un sentiment d'effroi et plus généralement à une paralysie.
Les médias ont été le théâtre privilégié du traitement affectif du terrorisme, relayant la parole des différents acteurs, affectés plus ou moins directement par ces événements : victimes, témoins, experts, politiques, historiens, chercheurs, policiers, juges, artistes. Au-delà de cette production sémantique, nous faisons l'hypothèse que les médias ont contribué à un déploiement sémiotique, voire " cosmologique », du terrorisme. Nous pouvons définir ce déploiement sémiotique comme la production de signes (linguistiques mais aussi 
iconiques et symboliques) dans le but d'assigner une signification aux attentats et à l'action même de leurs auteurs. Cette matière sémiotique offre alors une opportunité idéale pour enquêter sur un phénomène récent et pour lequel il existe peu de données tangibles.

\section{Saisir le terrorisme à partir de son traitement médiatique}

Le traitement médiatique d'événements terroristes, et plus généralement politiques, a suscité un vif intérêt de la part de la communauté scientifique, notamment depuis les années 1970. Différents travaux se sont intéressés ces dernières décennies aux rapports Les savoirs exprimés dans qu'entretiennent les médias les médias auraient ainsi avec les événements terrovocation à remettre sur pied les balises de l'ordre social et, plus particulièrement, le (re)découpage « entre ordre et désordre » social. ristes. Dès les années 1970, les médias sont accusés de jouer un rôle de catalyseur (voire de " coefficient multiplicateur $\left.{ }^{1} »\right)$ des sentiments (peur, effroi, angoisse) provoqués par le terrorisme ${ }^{2}$. Ils seraient ainsi responsables d'une banalisation du " terrorisme $e^{3}$ » en rendant ces événements spectaculaires, romantiques, esthétiques ou théâtraux ${ }^{4}$. Une lecture moins critique attribue néanmoins aux médias une capacité à agencer ou « encadrer » (framing) des récits porteurs de sens. Les discours médiatiques joueraient ainsi un rôle de cristallisation de l'opinion publique, en structurant des récits producteurs de « réalité objective ${ }^{5}$ ». Plus récemment, certains auteurs suggèrent que la production de récits « encadrants » (frames) est elle-même déterminée par un jeu complexe de relations avec les acteurs ${ }^{6}$. Même sous cette hypothèse permettant de penser les médias comme déterminés par des cadres (cognitifs, culturels, sociologiques...) individuels, ceux-ci demeurent des producteurs de stabilisations ${ }^{7}$. Ils vont ainsi participer à la construction de définitions stables d'objets diffus, en déployant des stratégies de captation, description, "monstration $^{8} »$. Mais, pour certains auteurs ${ }^{9}$, les médias vont également intervenir dans la construction d'oppositions stables entre systèmes de valeurs (bien/mal, Occident/Orient, français/étranger, citoyen/délinquant). Le propre du terrorisme serait ainsi de bousculer radicalement les marqueurs de la vie collective. Les savoirs exprimés dans les médias auraient ainsi vocation à remettre sur pied les balises de l'ordre social et, plus particulièrement, le (re)découpage « entre ordre et désordre » social ${ }^{10}$. Toutes ces hypothèses partagent un fond ontologique commun : le terrorisme est un fait. Il préexiste aux médias même si ceux-ci participent à son amplification ou à son détournement. Alors, le « terrorisme » n'est pas un objet médiatique en soi, même si son traitement n'est pas libre de conséquences sur un plan politique et idéologique.

L'enquête que nous proposons de restituer suggère une voie d'analyse différente. Nos résultats montrent que les médias contribuent pleinement à l'objectivation/l'instauration de la figure du «terroriste ». Le récit médiatique ne se limite pas à relater (plus ou moins intensément) un fait, mais l'instaure, le construit pleinement. C'est cette activité de mise en production du terrorisme que nous nommons « ontologie médiatique du ter-

\footnotetext{
1. Pierre Mannoni, Un laboratoire de la peur, terrorisme et media, Marseille, Hommes, 1992. 2. Friedrich Hacker, Terreur et Terrorisme, Paris, Flammarion, 1976 ; Jerry Mander, Four Arguments for the Elimination of Television, New York, Kill, 1977. 3. Élise Fréron, «La représentation médiatique du phénomène terroriste : quelques enseignements du cas nord-irlandais ", in Topique, vol. 2, n 83, 2003, pp. 135-147. 4. Didier Bigo, Daniel Hermant, "La relation terroriste ", in Études polémologiques, vol. 3, n 47, 1988 ; Christine Bonardi, Pierre Mannoni, « Terrorisme et Mass Médias », in Topique, n 83, pp. 55-72. 5. William Gamson, André Modigliani, «The changing culture of affirmative action », in Richard G. Braungart, Margareth M. Braungart (dir.), Research in Political Sociology, $n^{\circ}$ 3, 1987 ; Denis McQuail, Mass Communication Theory, New York, Sage, 2010 [1983]. 6. Dietram Scheufele, "Framing as a theory of media effects ", in Journal of Communication, $n^{\circ}$ 49, 1999, pp. $103-122$. 7. Ibid. 8. Aurélia Lamy, "Les spécificités du traitement médiatique dans l'urgence. L'exemple des attentats du 11 septembre 2001 ", in Communication et organisation, $n^{\circ}$ 29, 2006. 9. Daniel Hermant, "Violence politique, attentats et kamikazat : l'hypothèque du 11 septembre ", Cultures \& Conflits, $n^{\circ}$ 63, 2006, pp. 13-23. 10. Sophie Mijolla-Mellor, " Le terrorisme entre ordre et désordre ", in Topique, vol. 2, n 83, 2003, pp. 23-34.
} 
rorisme ». Cette ontologie médiatique cherche à nommer, définir, stabiliser le terrorisme, grâce notamment au déploiement de tout un travail de narration, de qualification et d'interprétation ${ }^{11}$. Nous mobilisons le terme d'ontologie au sens de Souriau (1943), c'est-à-dire dans un sens qui permet de saisir empiriquement comment différents êtres ou entités (ici le terroriste) s'instaurent dans des modes d'existence pluriels (ici nous faisons l'hypothèse d'un mode d'existence médiatique qui s'ajoute à d'autres modes d'existence du terroriste, notamment celui du droit).

\section{Représenter \\ les « ondes de choc » médiatiques}

Nous avons choisi de travailler sur les perceptions médiatiques des auteurs des faits, que les médias désignent comme étant « terroristes ». L'entrée par le concept de "terroriste " plutôt que par celui de " terrorisme " a un triple intérêt. D’abord, il permet de situer la question directement sur un plan ontologique dans la mesure où " terroriste » désigne un objet médiatique particulier et renvoie immédiatement à une personnification de l'événement. De même, nous avons constaté que les savoirs exprimés étaient bien plus riches et précis à l'heure de qualifier le "terroriste " plutôt que le «terrorisme ». Enfin, l'analyse du «terroriste » offre une opportunité idéale pour saisir les frontières et les stratégies discursives entre thématiques migratoires et terrorisme. Son traitement médiatique, renvoyant immédiatement à l'identité (géographique, culturelle, religieuse, sociologique...) du terroriste, permet de mettre en évidence les associations conceptuelles opérées par les médias. Nous supposons ainsi que le dévoilement de l'identité (médiatique) du «terroriste » est une bonne manière d'appréhender l'identité de l'« objet terrorisme ». Nous avons porté notre attention sur un échantillon de six titres fran-

çais (Le Monde, Le Figaro, L'Humanité, L'Express, La Croix, Libération) pour lesquels nous avons extrait 1852 articles traitant des attentats entre le $1^{\text {er }}$ janvier 2015 et le 31 janvier 2016, soit un corpus de plus de 5000 pages et 2,5 millions d'unités lexicales. Nous avons ensuite travaillé sur différents corpus (par titre de presse, par quinzaine de publication, par mois) afin de produire une analyse statistique en cohérence avec les questions traitées. Nous avons analysé ces données avec divers logiciels de traitement statistique et sémantique

Nous pouvons noter que les champs lexicaux de «l'étranger » ou encore de «l'émigration/immigration» sont présents de manière importante, apparaissant tout de suite après ceux relatifs au « conflit», au « combat » ou à l'u armée ».

(TROPES, DMI Tools, R, Textalyser) afin d'identifier la structuration lexicale des discours médiatiques ainsi que leur évolution dans le temps. Enfin, nous avons utilisé des outils de visualisation de données (Raw, D3JS) dans l'objectif de produire des résultats consistants et facilement interprétables.

Après avoir explicité comment les médias tendent à qualifier l'objet "terroriste ", nous exposerons trois résultats qui vont dans le sens d'une instabilité significative, voire parfois radicale, dans le traitement médiatique des attentats. Une fois ces résultats exposés nous serons en mesure de dessiner un certain nombre d'hypothèses interprétatives quant au mode d'existence du terrorisme.

\section{Comment les médias qualifient le «terroriste » ?}

Afin de saisir la manière générale avec laquelle les six titres de presse de notre échantillon se saisissent de la question, nous avons identifié les principaux termes linguistiques utilisés pour qualifier les terroristes ainsi que leurs modes d'action. Le tableau suivant recense les verbes, les adjectifs et les substantifs les plus utilisés. 
/// Figure 2 : Verbes, adjectifs et substantifs utilisés pour qualifier les terroristes (en nombre d'occurrences dans le corpus global).

\begin{tabular}{|c|c|c|c|c|c|c|c|}
\hline Vobus & Ocameares & Aducts & Oenmeates & Solutenth & Oreareses & Stbonethingel & Qenuness \\
\hline molvalinar & $\leq 2$ & Dane & $\mathbf{M}$ & Gheline & as & atust & 5 \\
\hline erceper & a & ackal & 15 & fors & 39 & teles & 5 \\
\hline exopiter & 9 & at an is & W & Gandirst & as & at at & 5 \\
\hline pulwaser & 9 & abpect & 11 & buas & 21 & weras & 4 \\
\hline hadr & 7 & asulfok & 11 & $\operatorname{actex}$ & 25 & inviluabee & 4 \\
\hline cerablest & 6 & entadan & W & $200 \mathrm{ar}$ & at & etes & 4 \\
\hline 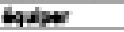 & 6 & Seet & 11 & howers & 21 & perver & 4 \\
\hline over & b & wand & 4 & heribow & i2 & suactiates & 4 \\
\hline achartive & 5 & du vyant & 10 & bas & 21 & fewernes & 4 \\
\hline ouv $\mathbf{y}$ & 5 & pues & 8 & frexcla & 22 & nartar & 4 \\
\hline aeerboviar & 4 & rasole & $\theta$ & ind lade & 23 & miles & 4 \\
\hline aedivese & 4 & Lentes & 8 & Easkae & 21) & tatile & 4 \\
\hline Gartide & 4 & MeN & s & dtrover & 21 & anative & 4 \\
\hline Devilaer & 4 & ulnivel & $\mathbf{3}$ & covraeda & 22 & leoder & 4 \\
\hline ageviser & 4 & Tabues & 6 & celeb & 25 & phe & 4 \\
\hline Eater & 4 & artwiaker & 6 & $f 4$ & 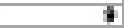 & mant & 4 \\
\hline denewiner & 4 & duegeres & 6 & eutstear & 9 & acentat & 4 \\
\hline nowler & a & cetiver & 6 & eevtie & 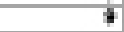 & nikerion & 4 \\
\hline bocher & I & volerain & 5 & tustringe & 5 & bukceer & 4 \\
\hline nober & a & lowesta & $\mathbf{s}$ & wlat & I & arabs: & 4 \\
\hline buedher & a & partase & 4 & cenean & t & allat & 4 \\
\hline bover & $\mathbf{a}$ & cread & 4 & bosmets & 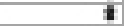 & 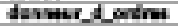 & 4 \\
\hline drager & 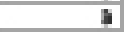 & uptacalaive & 4 & onguniaben & 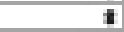 & ovet & $\mathbf{a}$ \\
\hline endeotion & $\mathbf{a}$ & cotriab & 4 & baitere & 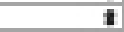 & fotile & $\mathbf{2}$ \\
\hline crober & a & reabers & 4 & alybing & t & - contwis & 2 \\
\hline bilyrer & 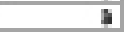 & and & 4 & ewbyr & 7 & fondurentaling & $\mathbf{2}$ \\
\hline epontir & $\mathbf{a}$ & andobing & 4 & alyurt & 7 & fdrecovinus & $\mathbf{3}$ \\
\hline wor & a & nivated & i & alifas & $\gamma$ & noesures & j \\
\hline becker & $\mathbf{a}$ & Ayter & $\mathbf{3}$ & foutses & 7 & Dequant & $\mathbf{2}$ \\
\hline \multirow[t]{13}{*}{ abrar } & a & sobitive & $\mathbf{2}$ & neased & 6 & vave & $\mathbf{2}$ \\
\hline & & Brovephont & 2 & covractina & 6 & abina & 2 \\
\hline & & violent & $\mathbf{2}$ & acheser & 6 & ata:Atur & $\mathbf{2}$ \\
\hline & & Nhankque & $\mathbf{3}$ & Bent & 5 & jose_berves & $\mathbf{3}$ \\
\hline & & apecal idquat & 2 & ento & 5 & Gadedor & 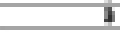 \\
\hline & & neperatl. & $\mathbf{2}$ & morocin & 5 & nain & $\mathbf{2}$ \\
\hline & & accasd & $\mathbf{2}$ & matare & 5 & ervi & $\mathbf{2}$ \\
\hline & & aldita & i & at & 5 & awh & i \\
\hline & & resubar & $\mathbf{2}$ & propiet & 5 & peapated & $\mathbf{3}$ \\
\hline & & illal & $\mathbf{2}$ & erfint & 5 & napivitin & $\mathbf{3}$ \\
\hline & & chadtan & $i$ & terratine & 5 & averita & i \\
\hline & & patrat & $\mathbf{3}$ & orlas & 5 & ahr & $\mathbf{2}$ \\
\hline & & & & & & eavopdea & $\mathbf{3}$ \\
\hline
\end{tabular}

/// Figure 3 : Principaux champs lexicaux identifiés (en nombre d'occurrences dans le corpus global).

\section{religion (154)droit (99)délit (85)conflit (71)armée (66)}

islam (64)homme (50)combat (49)famille (46)gens (46)politique (39)groupe_social (35)militaire (30) étranger (27)oeuvre (26)auteur (25)extrémisme (22)proche_orient (22)organisation (18)rrance (17)mammifêres (17)atrique (16)

afrique_du_nord (16)catastrophe (16)canidés (15)commerce (15)corps (15)émigration / immigration (15)enfant (14)trançals (13)comportement (12)

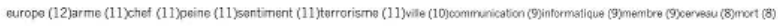

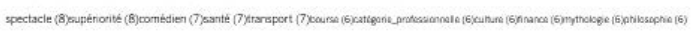

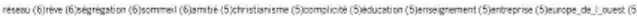


Nous pouvons constater une grande hétérogénéité des termes mobilisés pour qualifier « les terroristes ». Plus largement, nous avons identifié, grâce au logiciel TROPES ${ }^{12}$, les principaux univers de référence du corpus global constitué. Le champ lexical du religieux («religion », « islam ») est de loin le plus évoqué, bien avant les champs lexicaux normatifs, juridiques et les représentations politiques. Nous pouvons noter que les champs lexicaux de «l'étranger » ou encore de "l'émigration/immigration » sont présents de manière importante, apparaissant tout de suite après ceux relatifs au " conflit ", au « combat» ou à l'« armée ».

\section{L'instabilité des représentations médiatiques}

Un premier niveau d'instabilité radicale apparaît quant à l'identification sémantique des terroristes, oscillant entre qualificatifs d'intelligence supérieure et d'irrationalité totale, entre repères biographiques factuels et repères identitaires subjectifs, entre individuation/atomisation et tentatives synoptiques de généralisation sociale. Au total, nous avons pu identifier 8 axes d'antinomie sémantique dans le corpus global.

Ces axes d'antinomie générale se retrouvent également dans les sous-corpus par titre de presse. Les trois titres de presse qui déploient en leur sein le plus d'antinomies sont : Libération (6 axes), L'Express (6), Le Monde (4). Ceux qui en déploient le moins sont : Le Figaro (3), L'Humanité (2) et La Croix (1). Enfin, nous avons recensé en tout 85 articles qui comportent des représentations antagonistes des «terroristes ». Il est intéressant de noter que la tension « Français-Européen-Occidental/Étranger-Immigré-Réfugié » se retrouve dans 32 articles de presse.
Une deuxième instabilité temporelle permet d'étudier comment certains discours tendent à se dissiper alors que d'autres deviennent des repères représentationnels axiomatiques. Le graphique suivant met en évidence les " ondes de choc » médiatiques à travers la représentation du nombre d'articles consacrés, par chacun des titres, aux attentats ${ }^{13}$. Nous pouvons noter 5 grandes phases du mouvement médiatique. Une première liée à la réaction des médias face au premier attentat de janvier 2015, une deuxième de stabilisation progressive (mars, avril, mai), une troisième phase d'atténuation quasi complète (lors de l'été), une quatrième signalant le second attentat (novembre) et une cinquième représentant un nouveau pic de publication en janvier 2016 (un an après l'attentat contre Charlie Hebdo). Nous pouvons noter que le deuxième attentat a mis en production plus d'articles médiatiques que le premier. Le Monde est le journal qui a le plus publié d'articles sur la période. Nous pouvons également relever que le classement relatif du nombre de contenus médiatiques publiés par les six titres de presse n'est pas resté constant le long de la période, en particulier lors des trois pics de publication. Enfin, le taux de Un premier niveau d'instabilité radicale apparaît quant à l'identification sémantique des terroristes, oscillant entre qualificatifs d'intelligence supérieure et d'irrationalité totale, entre repères biographiques factuels et repères identitaires subjectifs, entre individuation/atomisation et tentatives synoptiques de généralisation sociale. décroissance du volume de production médiatique a été plus important après le premier attentat qu'à la suite du second.

L'instabilité sémantique se double d'une instabilité temporelle : latent, mais jamais absent, le fait " terroriste " est construit à travers les médias avec la temporalité de l'explosion soudaine, dont l'onde de choc se dissipe avec le temps. Cette présence sous forme d'absence contribue, nous 
Source: Base de données constituée par les auteurs à partir d'une extraction d'articles de presse des journaux Le Monde, La Croix, Libération, L'Humanité, L'Express et le Figaro. A noter que les couleurs ne renvoient pas à des significations particulières mais assurent une meilleure visualisation de l'information.

semble-t-il, ainsi à l'instabilité de l'objet médiatique. Cette analyse quantitative ne nous permet cependant pas d'identifier avec précision l'architecture des récits sémiotiques dans sa temporalité. Pour ce faire, nous avons voulu représenter les dynamiques d'éclosion, évolution et atténuation des discours médiatiques au cours du temps. Le Stream Graph suivant décrit l'évolution dans le temps des champs lexicaux utilisés par les médias pour décrire les terroristes. Là aussi, nous avons décomposé le corpus global en sous-corpus par quinzaine de publication. Il permet d'identifier la place relative de chaque champ lexical détecté dans chacun des sous-corpus par titre de presse.

Le premier constat est que les champs lexicaux sont extrêmement variables dans la durée. Certains champs sont étroitement corrélés au surgissement des événements et à leur domestication par les acteurs institutionnels politiques, juridiques et sécuritaires (" conflit », " combat », 
" droit », " délit »). On retrouve ici de nombreux articles à caractère descriptif, relatant les événements ou le profil des suspects (" groupe social ", " auteur », "famille »).

De nombreux champs lexicaux vont connaître une trajectoire cyclique et vont apparaître (ou réapparaître), de manière plus ou moins prononcée, une fois le pic médiatique retombé. C'est notamment le cas des champs lexicaux de la "religion " (ici "religion » et « islam »), de l'" armée », du " délit » ou du "droit ». Ils correspondent à des phases médiatiques d'analyse ou de réflexion. Il est intéressant de noter l'existence de deux séquences temporelles à ce niveau-là. La première se situe entre avril et juin et correspond, après vérification au sein des sous-corpus, en grande partie à des articles d'analyse, de débat et d'interprétation. Des acteurs autres que médiatiques sont convoqués (experts, chercheurs-universitaires, artistes) pour exprimer leur perspective de l'événement. La deuxième phase, aux alentours de l'été, montre bien une tendance à la monopolisation des discours à partir des perspectives juridiques et sécuritaires de l'événement (champs du « délit » et du «droit » prépondérants). Un exercice de va-etvient avec les articles de notre base de données confirme bien que ceux-ci sont dédiés en grande proportion à l'enquête juridique.

Il nous semble également important de signaler l'hétérogénéité du traitement médiatique entre les deux d'attentats. Certains récits vont avoir tendance à s'effacer (" extrémisme », " politique ») lors du second événement, alors que d'autres vont émerger de manière plus ou moins exponentielle (" immigration », " étranger », " militaire », « armée »). Ce traitement différencié des deux attentats reflétant potentiellement une différence radicale dans la manière d'être affecté par les deux événements. Dans un cas (premier attentat), la focale politique, religieuse, juridique est prépondérante (reflétant notamment une grande quantité d'articles dédiés aux débats publics autour de la « liberté d'expres- sion ", la "laïcité », les " mesures politiques » à prendre...). Dans l'autre, la focale est déplacée sur des thématiques militaires et des problématiques migratoires (là aussi étroitement liées aux débats publics concomitants sur les "réfugiés", la « crise des migrants », mais aussi sur « l'intervention militaire contre Daesh »). La manière dont les médias vont qualifier les terroristes va changer là aussi radicalement dans la mesure où le second attentat va faire émerger un grand nombre de champs lexicaux liés à l'organisation (" professionnelle », " structurée », " stratégique ») des équipes. Les terroristes sont moins des « loups solitaires » (d'où la présence significative du champ lexical des mammifères lors du premier attentat), des "individus isolés ", que de véritables « entreprises », " projets » et autres « commandos » bien «structurés ».

Le surgissement des thématiques migratoires va s'opérer dans les traitements médiatiques de deux manières différentes.

D'abord, nous avons observé Les terroristes sont moins que, lors du premier atten- des «loups solitaires» tat, peu d'articles vont sug- (d'où la présence significative gérer des liens entre "ter- du champlexical des roristes » et « immigration » mammifères lors du premier (nous en avons recensé 19 attentat), des «individus contre 75 pour le second). isolés», que de véritables L'identité des «terroristes » "entreprises», «projets » et est généralement abor- autres «commandos » bien dée de manière relative- "structurés». ment factuelle : «français », " urbains », « nés dans des communes françaises », " nés en banlieues », entre autres. Cette manière de traiter l'identité géographique des terroristes va changer lors du second attentat: les origines identitaires des terroristes sont beaucoup plus explicites ( franco-algérien », « belgo-marocain », « syrien », « musulman », « d'origine syrienne »...). Un accent plus prononcé est mis sur les trajectoires spatiales empruntées (Irak, Syrie, Yémen, Turquie). Plus généralement, les thématiques migratoires et terroristes vont subir des traitements communs au sein 
Source: Base de données constituée par les auteurs à partir d'une extraction d'articles de presse des journaux Le Monde, La Croix, Libération, L'Humanité, L'Express et le Figaro. A noter que les couleurs ne renvoient pas à des significations particulières mais assurent une meilleure visualisation de l'information.

des mêmes articles ou au sein des mêmes éditions (33 articles abordant de manière concomitante « terrorisme » et « immigration/réfugiés » contre seulement 3 pour le premier attentat).

\section{Le terrorisme comme pratique de désémantisation}

Si l'on adopte l'approche développée par les théoriciens de l'apprentissage socialement situé ${ }^{14}$, nous pouvons voir se dessiner de véritables « commu- nautés sémantiques » derrière la création de ces champs sémantiques particuliers à chaque titre de presse et communs à l'ensemble des titres. Ces communautés peuvent être appréhendées sur le modèle des communautés épistémiques et de pratiques $^{15}$, c'est-à-dire de véritables "lieux de la culture $^{16}$ » qui situent les connaissances dans un espace expérientiel précis, lié à certaines pratiques. Cela implique donc que la perception, l'expérience et la sémantisation du «terroriste » varient, et parfois considérablement, d'une communauté sémantique à l'autre, tout en gardant un fond commun 
qu'il est sans doute important de questionner. Les différentes communautés sémantiques délimitent aussi des communautés politiques dans lesquelles certaines pratiques et certaines définitions ont cours et d'autres sont exclues (l'assignation à un champ sémantique est un faire et participe à la construction de définitions de "terroriste " et de «terrorisme »). C'est la coexistence dans le même paysage médiatique de toutes ces pratiques et de toutes ces définitions qui contribue fortement à stabiliser l'instabilité du terrorisme.

La pratique de sémantisation/agentivité peut être assimilée à une sorte de " coloriage », c'està-dire qu'à l'intérieur d'une certaine communauté sémantique certaines pratiques et certains champs lexicaux sont teintés d'une valence " terroriste ». L'agentivité réside ici dans la capacité de transporter un nombre de plus en plus important d'éléments d'un champ lexical et d'un répertoire de pratiques vers cet ensemble, au profil indéfini, de ce qui fait «terroriste » ou « terrorisme ». Cette agentivité fait écho à l'agentivité épistémique dont a fait preuve le législateur dans sa manière de définir de plus en plus largement les qualifications des actes "terroristes ». Ce coloriage fonctionne de manière à attribuer à certains éléments d'un champ lexical une certaine nuance, un " air de famille $e^{17}$ ". L'instabilité médiatique apparaît ici comme l'effet de cette modalité de sémantisation particulière qui est la capacité à renvoyer un nombre très important d'éléments hétéroclites vers une seule nuance, de les teindre d'un seul colori, celui du terrorisme; en quelque sorte, le «terrorisme » est ce vers quoi on renvoie quelque chose et jamais un élément définissable en soi.

Cette capacité est le fondement épistémique de l'instabilité et de l'incertitude expérientielle du terrorisme et correspond à l'idée que véhiculent les médias que tout peut être a priori sujet de/du « terrorisme " (incluant " jeunes ", «barbus ", " radical », « ami », « frère », « immigré », « réfugié », etc.). Finalement, par sa modalité propre de fonctionnement - ce renvoi vers quelque chose qui donne un
" air de famille » en lieu et place de renvoyer vers une signification stable -, ce régime de sémantisation produit une perte sémantique qui transforme la pratique en un geste de "désémantisation » ou de « désignification ». Désémantisation car le coloriage qui s'opère avec le renvoi d'une pratique ou d'un élément lexical vers la nuance "terroriste " finit par ôter toute intelligibilité à l'acte de définition du terrorisme. Plus l'agentivité sémantique est importante, plus il sera possible d'incorporer un nombre de plus en plus important de champs sémantiques à l'intérieur du réservoir lexical des communautés. Ainsi, aucun champ lexical ne peut être épargné a priori et risque constamment de se retrouver incorporé dans l'ensemble des champs colonisés par le « terrorisme ».

\section{Le terrorisme comme absence latente}

Pourtant, une logique semble à l'œuvre dans le régime de sémantisation qu'opère le " terrorisme ». On constate une absence flagrante des champs sémantiques ayant trait à la féminité et aux femmes, ainsi qu'à tout référent historique comme colonies, colonisation, colonial, coloniaux. Ces absences suggèrent une inscription du « terrorisme » dans le registre de ce qu'il n'est pas. Comme l'explique Jasbir Puar ${ }^{18}$, les enjeux de (dé)stabili- C'est dans le même sens sation définitionnelle du que l'on peut lire l'absence «terrorisme » s'étendent significative du champ lexical sur l'ensemble des sites colonial et la rupture qui de tension politique, en en est faite avec l'histoire. particulier sur ces lieux de tension que sont les rapports à la racialisation, au genre et à la sexualité. La masculinité hétérosexuelle apparaît en toile de fond de cette procédure de désémantisation. Si à peu près tout peut être assigné au « terroriste » (figure 4), l'absence du champ lexical ayant trait à la féminité réitère et réifie une exclusivité du masculin et des hommes 
dans les champs lexicaux incorporés. En fait, le terrorisme est une histoire d'hommes. C'est dans le même sens que l'on peut lire l'absence significative du champ lexical colonial et la rupture qui en est faite avec l'histoire. Cette absence fait écho à la surreprésentation du champ lexical de la religion et de l'islam, et à son association avec celui de l'immigration. En tant qu'écho à cette surreprésentation, l'absence du champ lexical colonial renforce l'idée d'une a-temporalité du « terrorisme ». Cette absence suggère un " terrorisme » à saisir dans sa dimension a-historique, oublieux de l'histoire longue des confrontations politiques et épistémiques que cet espace culturel sexuellement, racialement, socialement, spirituellement marqué qu'est l'Occident, a nourri tout au long de l'histoire.

\section{Le mode d'existence du «terroriste »}

Dans la tradition philosophique, le concept de " modes d'existence » renvoie à l'idée d'un pluralisme ontologique. Il s'agit ainsi de prendre au sérieux les êtres qui traditionnellement échappent à une définition basée uniquement sur les registres

Plus spécifiquement, nous avons retenu que lorsque l'onde de choc médiatique s'atténue, les champs lexicaux du délit et du droit prennent

la relève. de la substance (comme dans une conception cartésienne, par exemple), de la conscience, de la matière... Pour Étienne Souriau $^{19}$, il existe en effet plusieurs modes d'existence permettant de saisir des êtres aussi divers que des objets techniques, des entités religieuses ou encore des êtres de fiction... Ces « êtres ", " choses » ou " objets ${ }^{20}$ » ne peuvent être saisis correctement (c'est-à-dire dans toute leur étendue) à travers un monisme ontologique, mais nécessitent que l'on prenne en compte (et que l'on décrive précisément) dans quels régimes d'objectivation ou de «véracité » ils s'inscrivent. Ces modes d'existence fonctionnent ainsi comme de véritables catégories d'existence.

Une analyse du phénomène " terroriste » à travers cette grille de lecture offre trois types d'opportunités. D'abord, il permet de prendre au sérieux l'hypothèse qui consiste à dire que le « terroriste » est un être qui s' « instaure » (pour reprendre le terme utilisé par Souriau) pleinement dans les médias. Et, plus précisément, cet être se donne à voir grâce aux différentes traces médiatiques qu'il génère et que nous avons sondé sémiotiquement. Notre enquête permet de suggérer qu'il existe en effet bel et bien un mode d'existence médiatique du terroriste, dans la mesure où son régime ontologique est soumis à des instabilités, des controverses et surtout à des tentatives d'objectivation. Or, selon Bruno Latour ${ }^{21}$, c'est bien ces caractéristiques qui définissent un mode d'existence (notamment celui du droit, ou de la science, que Latour a amplement étudié22).

Ensuite, l'idée d'un pluralisme ontologique permet de penser la possibilité d'une existence distribuée de l'objet terroriste sur plusieurs modes. Car le pluralisme ontologique ne signifie pas un enfermement des entités dans des registres stricts. L'instabilité temporelle observée a permis de montrer que certains récits entourant les « terroristes » tendaient à se dissiper alors que d'autres tendaient à éclore. Plus spécifiquement, nous avons retenu que lorsque l'onde de choc médiatique s'atténue, les champs lexicaux du délit et du droit prennent la relève (figure 6). Ce résultat suggère donc un deuxième mode d'existence à travers lequel l'objetterroriste se donne et qui est celui du droit. Car le droit participe pleinement, grâce à un travail d'enquête, de consolidation des preuves, de qualification des actes (" actes de terrorisme ", " association de malfaiteurs ", " entreprise terroriste »), à l'objectivation du «terroriste ». L'onde de choc se caractériserait ainsi par une compétition entre des 
Source: Base de données constituée par les auteurs à partir d'une extraction d'articles de presse des journaux Le Monde, La Croix, Libération, L'Humanité, L'Express et le Figaro. A noter que les couleurs ne renvoient pas à des significations particulières mais assurent une meilleure visualisation de l'information.

modes d'existence distincts cherchant à stabiliser l'être-terroriste. Dès lors, toute la question est de savoir quel mode d'existence dominerait, à long terme, l'autre. Car, et c'est là qu'apparaît la troisième opportunité, les médias ne contribuentils pas à sculpter des êtres imaginaires au sens de Souriau, c'est-à-dire des êtres "fragiles », "inconsistants », "transitifs » et qui ne peuvent "pas être rangés dans un monde stable ${ }^{23}$ "? Le terroristemédiatique a, en effet, besoin d'exister à travers une série de qualifications hétérogènes, des contributions d'experts, policiers, universitaires, journalistes, témoins... Or ces contributions sont amplement nourries par les affects qui lient tous ces acteurs aux événements. À l'opposé, le terroristejuridique (au sens d'entité définie sur le mode d'existence du droit) n'est pas conditionné au volume d'affects (bien au contraire, comme l'a démontré Latour ${ }^{24}$ pour les êtres du droit).

Bien que le terroriste-médiatique bénéficie d’un statut ontologique fragile et inconstant, il reste un être de «remote presence ${ }^{25}$ » et donc demeure puissant, notamment politiquement. En effet, il y a bien aussi un mode d'existence politique du «terroriste ». La preuve en est la volonté des pouvoirs publics, immédiatement après les attentats, de vouloir déchoir les individus présumés de terrorisme de leur nationalité française. Cette décision 
est l'essence même d'une ontologisation politique, par le biais d'une décision gouvernementale, modifiant la définition même de l'identité citoyenne du présumé. On pourrait donc dire, en analysant cette réaction politique, que même dans le cas d'un mode d'existence médiatique inconstant, celuici a la capacité de bouleverser les cadres (moraux, normatifs, philosophiques) des modes d'existence juridiques et politiques du « terroriste ».

\section{Conclusion}

Le philosophe Peter Slotedijk a proposé récemment une analyse du terrorisme à partir d'une grille de lecture qu'il appelle "sphérologique ". Le terrorisme fonctionnerait ainsi comme une production immunologique et dont l'existence ne serait pas liée à des actes que l'auteur allemand qualifie d'" isolés » et commis " par de simples criminels». Le terrorisme sert bien à "quelque chose» et en l'occurrence à donner de la "cohérence " aux ensembles sociaux occidentaux. Pour Sloterdijk, " en un mot, dans la perspective de la realpolitik, si le terrorisme n'existait pas, il faudrait l'inventer ${ }^{26}$ ". Il faut ainsi un "élément de paranoïa » pour que les sociétés occidentales puissent actualiser leurs systèmes immunologiques ${ }^{27}$, notamment symboliques et rituels. Enfin, pour Sloterdijk, le propre du terrorisme moderne (qu'il soit terrorisme d'état ou terrorisme distribué) est d'être un "atmo-terrorisme $e^{28}$ ». II ne s'attaque pas seulement aux individus, mais vise leur atmosphère, c'est-à-dire leurs systèmes de protection immunologiques. Or le propre du terrorisme contemporain réside bien dans sa capacité à être "spectaculaire ${ }^{29}$ ", à produire une mise en scène. Il s'attaque ainsi à l'atmosphère des sociétés occidentales, les médias, comme lieux privilégiés dans la production d'affects et de référents symboliques et politiques ${ }^{30}$. Se dessine alors l'image d'une nouvelle grande architecture ontologique tournée vers l'intérieur et qui passe progressivement par la définition puis l'exclusion de nouveaux « autres ». Notre enquête suggère l'existence d'une nouvelle fabrique des " autres " sous la forme d'une production d'altérités qu'est l'espace médiatique post-attentats. Ce système médiaticoimmunologique, selon nos résultats, dispose d'une caractéristique principale : elle est distribuée selon une forme éclatée et circulaire. Les représentations médiatiques, comme nous l'avons vu notamment au travers des axes antinomiques identifiés, apparaissent comme un périmètre circulaire. Le « terroriste » est une figure qui nous pose problème ontologiquement. Il ne peut être en dedans de la sphère. Il doit être extériorisé. Or sa qualification binaire (coupable/non coupable) sous l'angle de la criminalité et du droit ne suffit apparemment pas à faire tenir la sphère collective agressée. D'où la nécessité d'ériger des systèmes de qualifications à deux niveaux (le terroriste est à la fois loup et agneau, mécréant et fou de Dieu, français et étranger...). On se protège ainsi de tous les côtés, en bâtissant des protections immunologiques symboliques, circulaires-sphériques et distribuées. Cependant, notre enquête nous a amenés aussi à identifier certaines marges de savoirs où tout l'enjeu consiste non pas à extérioriser un "ennemi " (production vaine) mais, bien au contraire, à l'intérioriser (au sens de le rendre pleinement conscient) jusqu'au point où il permette de comprendre pourquoi nous fabriquons des ennemis. 